\title{
Indications for PET/CT in the Head and Neck
}

\author{
Vikas Agarwal, MD ${ }^{\mathrm{a}, *}$, Barton F. Branstetter IV, MD ${ }^{\mathrm{a}}$, \\ Jonas T. Johnson, $\mathrm{MD}^{\mathrm{b}}$ \\ a Department of Radiology, University of Pittsburgh Medical Center, 200 Lothrop Street, \\ Pittsburgh, PA 15213, USA \\ ${ }^{\mathrm{b}}$ University of Pittsburgh School of Medicine, Eye and Ear Institute, 200 Lothrop Street, \\ Suite 500, Pittsburgh, PA 15213, USA
}

Head and neck cancer accounts for approximately 3\% to 5\% of all new cancer diagnoses in the United States with 40,000 new cases diagnosed each year [1]. These figures encompass cancers arising from the lip, oral cavity, nasal cavity, paranasal sinuses, pharynx, and larynx, of which the vast majority $(90 \%$ to $95 \%)$ are squamous cell carcinomas arising from mucosal linings of the upper aerodigestive tract. Other, rare cancers that may involve the head and neck include salivary tumors, thyroid cancers, lymphoma, and melanoma.

Early diagnosis and accurate staging are essential for treatment planning and can strongly influence prognosis. Likewise, early identification of tumor recurrence can often be treated with additional surgery or re-irradiation. A combination of history, physical exam, endoscopy, and tissue sampling has historically been the mainstay of diagnosis and staging. The use of crosssectional imaging (CT and MRI) has greatly improved staging and monitoring for disease recurrence. Small metastases and/or early recurrent disease, however, can still be missed [2].

Functional imaging with 18F-fluorodeoxyglucose (FDG) positron emission tomography (PET) can be used to fill these gaps and improve characterization of both primary tumors and metastatic disease. PET has been shown to be effective in the diagnosis of many different types of cancer, including head and neck squamous cell carcinoma (HNSCC) [3]; however, the poor spatial resolution of PET and the lack of anatomic landmarks can make exact localization of disease difficult. In addition, normal

\footnotetext{
* Corresponding author.

E-mail address: agarwalv@upmc.edu (V. Agarwal).
} 
anatomic structures may show variable FDG uptake causing errors in interpretation (Fig. 1). As a result, combined PET/CT scanners have become available that allow for both anatomic and functional characterization of disease at the same time.

$\mathrm{PET} / \mathrm{CT}$ has revolutionized the staging and surveillance of patients with HNSCC by allowing more accurate staging, more focused treatment modalities, earlier detection of recurrent disease, and identification of incurable disease to avoid overtreatment. The integration of PET and CT is more accurate than either modality alone for the detection of malignancy in the head and neck [4]. There is improved localization of abnormalities, better differentiation of therapeutic changes from residual disease, and improved assessment of tumor extent [4-7]. There are, however, several potential drawbacks to PET/CT imaging for cancers of the head and neck. Compared with conventional imaging modalities, PET/CT is more expensive. In addition, some cancers of the head and neck do not uniformly accumulate FDG, and potential false positives are numerous [8].

\section{Staging of HNSCC}

Squamous cell carcinomas of the head and neck are staged according to the American Joint Committee on Cancer (AJCC) Staging Manual. Information regarding the primary tumor size and extent $(\mathrm{T})$, regional nodal involvement $(\mathrm{N})$, and distant metastases $(\mathrm{M})$ are synthesized to tabulate
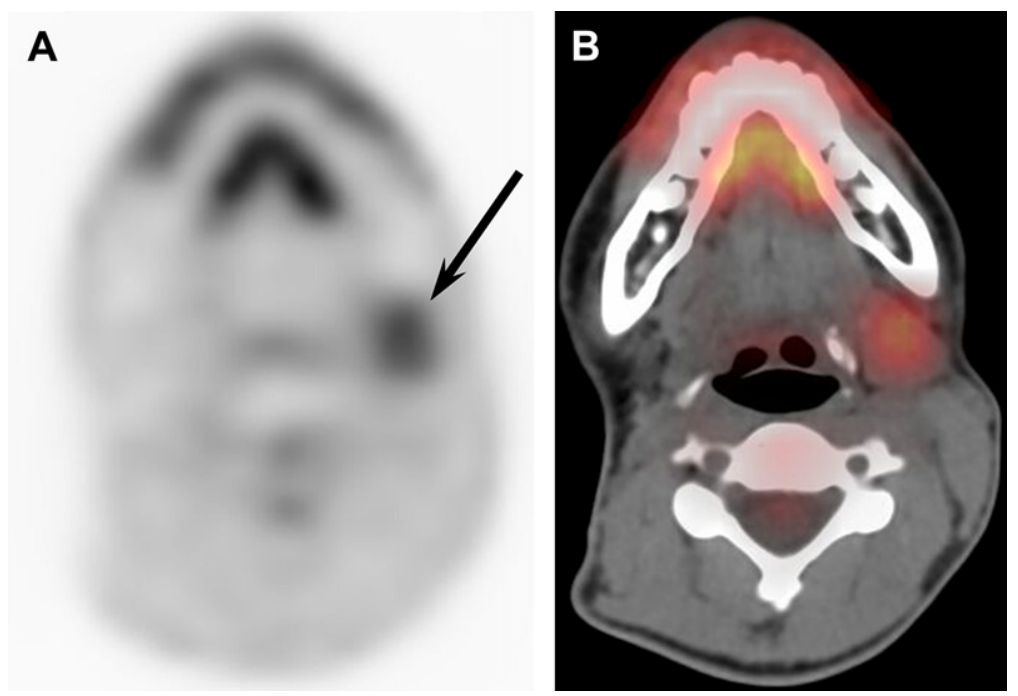

Fig. 1. False-positive PET suggests nodal disease. (A) Axial PET image is worrisome for left Zone II lymphadenopathy (arrow). (B) Fused PET/CT image correctly localizes the FDG uptake to the submandibular gland, avoiding a false-positive interpretation. 
a stage allowing for more precise treatment planning and accurate prognostic information [9].

A combination of history, physical examination by an experienced clinician, endoscopy and tissue sampling with fine needle aspiration (FNA), or core biopsy has been the mainstay for diagnosis and staging of HNSCC, but imaging has now become an integral part of the work-up. Imaging allows for a more clear assessment of degree of infiltration by the primary tumor, involvement of lymph nodes, and detection of distal metastases or second primary tumors. This ultimately improves diagnosis and staging and allows for optimal treatment planning and accurate posttreatment follow-up.

\section{T stage}

Criteria for defining the T stage in patients with HNSCC vary slightly according to the site of primary tumor. Generally speaking, accurate and complete assessment of the $\mathrm{T}$ stage requires knowledge not only of the size of the primary lesion but also the depth of invasion and involvement of surrounding structures. Imaging with contrast-enhanced CT or MRI has become an essential complement to physical examination and biopsy when assessing the $\mathrm{T}$ stage before initiating treatment $[7,10]$. CT can provide information regarding the depth of local invasion and infiltration into adjacent structures. In most cases, CT and MRI provide similar information and the decision regarding which modality to use is generally based on a radiologist's personal preference. MRI is, however, superior to CT for several specific clinical questions such as presence or absence of perineural spread or invasion of bone marrow (see the article by Drs. Ahmad and Branstetter, elsewhere in this issue).

Multiple studies have shown PET to be highly accurate in detection of primary tumors [11,12]; however, PET has not been shown to be superior to conventional cross-sectional imaging modalities (CT and MRI). Furthermore, metabolic information alone is not sufficient to provide the information required to accurately and completely stage the primary lesion. Combined PET/CT scanning supplements the functional information with anatomic information from a contrast-enhanced CT. To date, PET/CT has, however, not been shown to be superior to standard anatomic imaging modalities in evaluation of the initial $\mathrm{T}$ stage of the primary tumor [13]. Therefore, only patients who are at substantial risk of nodal or hematogenous metastases (T3 or T4) should undergo evaluation with staging $\mathrm{PET} / \mathrm{CT}$.

\section{$N$ stage}

The spread of disease to regional lymph nodes is the most important prognostic factor in squamous cell carcinoma of the head and neck. With the exception of primary nasopharyngeal cancers, staging for nodal 
metastases in HNSCC is uniform under the AJCC system. Characteristics that need to be evaluated include the total number of nodes involved, the size of the involved nodes, and the location (unilateral or bilateral and specific levels) and morphology of the nodes involved.

Compared with physical exam, imaging clearly improves the detection of regional lymph node metastases with CT outperforming MRI [14,15]; however, no definite criteria exist for identifying metastatic cervical lymph nodes on conventional imaging modalities. Size criteria are the most commonly reported. Smaller thresholds for lymph node diameter result in higher sensitivities at the expense of specificity. In HNSCC, there is high propensity for small lymph nodes to harbor tumor. Even at a commonly accepted threshold diameter of less than $10 \mathrm{~mm}$, most malignant nodes in patients with HNSCC would be missed by conventional imaging modalities thereby understaging patients [16]. Central necrosis, when present, has been shown to be specific for malignancy [17]. It is, however, a later marker of metastatic adenopathy seen primarily in lymph nodes greater than $20 \mathrm{~mm}$ in diameter, thereby making it an insensitive indicator of disease on CT [16]. Other criteria used include contrast enhancement with lymph nodes that have a greater degree of enhancement more likely to harbor tumor, and morphology with rounded shape more worrisome than reniform shape.

PET has been shown to be superior to conventional imaging modalities for the detection of regional nodal metastases [18]. The increased glucose metabolism can be identified in lymph nodes infiltrated by tumor cells regardless of size criteria. The utility of PET alone is, however, limited by spatial resolution, which makes anatomic localization difficult especially given the complex anatomy within the head and neck. PET/CT bridges this gap by combining metabolic and anatomic information and allows for the most accurate assessment of regional nodal metastases (Fig. 2). In a study of 20 patients with HNSCC, Schwartz and colleagues [19] found $\mathrm{PET} / \mathrm{CT}$ had an increased sensitivity $(96 \%)$ for detection of nodal disease compared with CT alone $(78 \%)$. Similar studies have shown that PET/CT significantly alters TNM scores in patients with HNSCC by identifying nodal disease not apparent by CT, MRI, clinical exam, or combination thereof. Koshy and colleagues [20] found that PET/CT altered the TNM score in $36 \%$ of 36 patients compared with CT alone, while Ha and colleagues [21] found that PET/CT altered the TNM score in $31 \%$ of 36 patients compared with either CT or MRI. In both of these studies, 7 of the 36 patients $(19 \%)$ had changes to their $\mathrm{N}$ status based on the information garnered from PET/CT but not identified on conventional imaging. Goerres and colleagues [22] found PET/CT altered the TNM score in $15 \%$ of 34 patients compared with clinical exam, CT, and chest radiography (in $7 / 34$ patients [21\%], the $\mathrm{N}$ stage changed). PET/CT is therefore the modality of choice when evaluating HNSCC patients for nodal metastatic disease. 

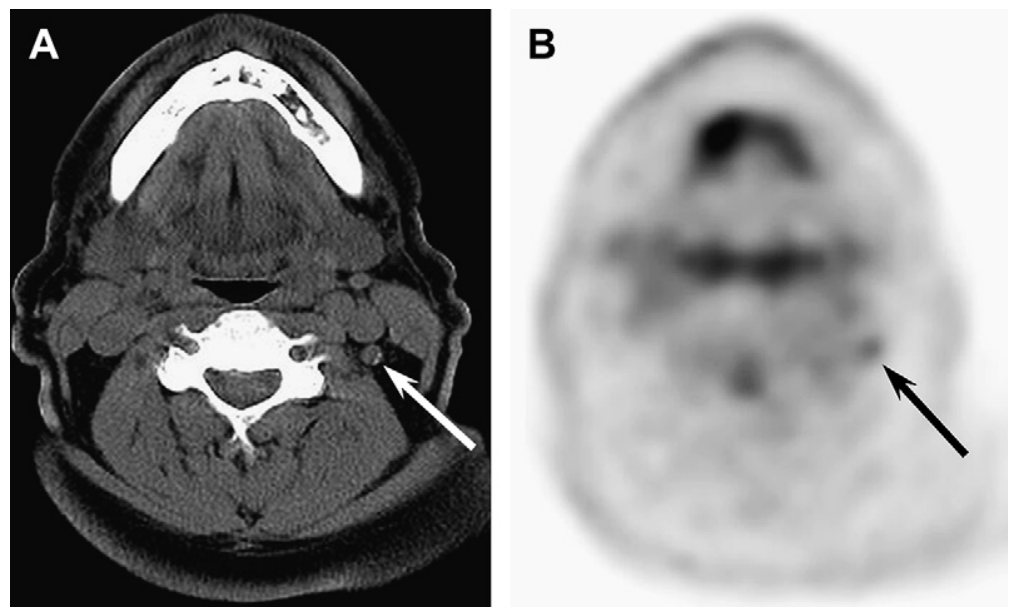

Fig. 2. False-negative CT overlooks nodal disease. $(A)$ On axial unenhanced CT, the 4-mm lymph node (arrow) is too small to be suspicious by size criteria alone. Tiny calcific foci and a rounded configuration are clues to the true diagnosis. $(B)$ Increased FDG uptake (arrow) correctly suggests malignancy (metastatic papillary thyroid carcinoma).

\section{$M$ stage}

Approximately $10 \%$ to $15 \%$ of patients with primary HNSCC will have distant metastases. The most common sites of involvement are the lungs, followed by the liver and skeletal system. The presence of distant metastases may indicate a need for additional therapeutic measures, whereas overlooked distant metastases can lead to inappropriately aggressive treatment $[23,24]$. In most patients, distant metastatic lesions will be clinically silent. The following is a list of subsets of patients with HNSCC in whom screening for distant metastases would be indicated [25].

- $\geq 4$ lymph nodes metastases

- Bilateral lymph node metastases

- Lymph node metastases $\geq 6 \mathrm{~cm}$

- Zone IV lymph node metastases

- Recurrent HNSCC

- Second primary tumor

Previous screening tests such as chest radiography, serum alkaline phosphatase, and liver function tests have been shown to be insensitive for the detection of distant metastases [26]. Bone scans are of extremely low yield, especially in asymptomatic patients. Chest $\mathrm{CT}$ is better than plain radiography but will still miss a significant number of thoracic metastases [23].

PET has previously been shown to improve detection of occult distant disease for other primary cancers including lung, esophagus, and lymphoma. Studies have showed similar success for patients with primary 
HNSCC [15,22,27]. Limitations of poor spatial resolution and lack of anatomic detail on PET imaging are overcome by integrated PET/CT (Fig. 3). Whole body PET/CT is more accurate than PET alone or traditional cross-sectional modalities for identification of distant metastases. Goerres and colleagues [22] showed that PET/CT found distant metastases or second primaries in $24 \%$ of their patients newly diagnosed with SCC of the oral cavity initially screened by clinical exam, CT, and chest x-ray. Similarly, Ha and colleagues [21] found that the TNM score was altered in $31 \%$ of their patients with previously untreated HNSCC initially staged with CT or MRI. PET/CT allows for better characterization of equivocal abnormalities on CT by PET and for more accurate anatomic localization of abnormalities on PET by CT [4]. With greater sensitivity than conventional modalities for the detection of metastatic lesions, PET/CT is the modality of choice for staging of patients with advanced HNSCC.

\section{Unknown primary tumors}

Patients with HNSCC sometimes present clinically with an enlarging neck mass for which FNA reveals metastatic SCC. In most cases, the primary tumor will be found by physical exam, cross-sectional imaging, or endoscopic evaluation. Despite these efforts, $2 \%$ to $9 \%$ of patients presenting with nodal metastases will have an occult primary tumor $[13,28]$. In these cases, the primary tumor may be too small $(<5 \mathrm{~mm})$ to be seen on endoscopy and imaging, or be in a location that may escape notice such as the tonsillar crypts. Likewise, cutaneous primary tumors and tumors outside the head and neck may be overlooked in the customary work-up.

When the primary tumor is found, the patient can be afforded the tissue sparing offered by modern focused radiation therapy techniques. Failure to find the primary relegates the patient to radiation of the entire upper aerodigestive tract, with the attendant morbidity of xerostomia and fibrosis.

Evaluation of patients with unknown primaries traditionally consists of physical exam, panendoscopy (orophayrnx, hypopharynx, nasopharynx,

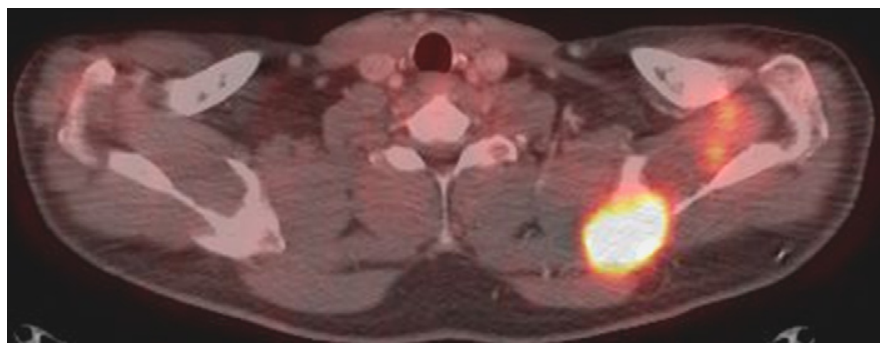

Fig. 3. False-negative CT overlooks metastatic disease. PET/CT shows left scapular bone metastases that was overlooked on conventional CT in a patient with follicular thyroid carcinoma. The CT component is still needed to adequately assess lesion location. 
larynx, and upper esophagus), anatomic imaging with CT and/or MRI, and blind tongue base biopsy. Numerous studies have addressed the ability of PET to identify primary locations after traditional work-up [29-31]. A meta-analysis by Rusthoven and colleagues [32] showed that PET was able to recognize a primary tumor after negative conventional work-up in one quarter of patients with an overall sensitivity and specificity of $88 \%$ and $75 \%$, respectively. In a similar study by Miller and colleagues, [31] PET was able to locate the primary tumor in $31 \%$ of patients studied with confirmed squamous cell carcinoma of the cervical lymph nodes and no visible primary tumor. PET was, however, negative in four patients in whom the primary tumor was discovered during routine panendoscopy. PET/CT has not been studied extensively, but is presumed to perform at least as well if not better than PET alone (Fig. 4). A small series found that PET/CT helped identify more primary tumors $(33 \%)$ than CT $(18 \%)$ or PET $(24 \%)$ alone [33]. Despite these promising numbers, there were still false negatives with $\mathrm{PET} / \mathrm{CT}$, where the primary tumor was identified by other diagnostic procedures. Therefore, PET/CT is a supplement, not a substitute, for endoscopy and biopsy in the setting of an unknown primary.

\section{The NO Neck}

The spread of disease to lymph nodes is the most important prognostic factor in squamous cell carcinoma of the head and neck. It is estimated
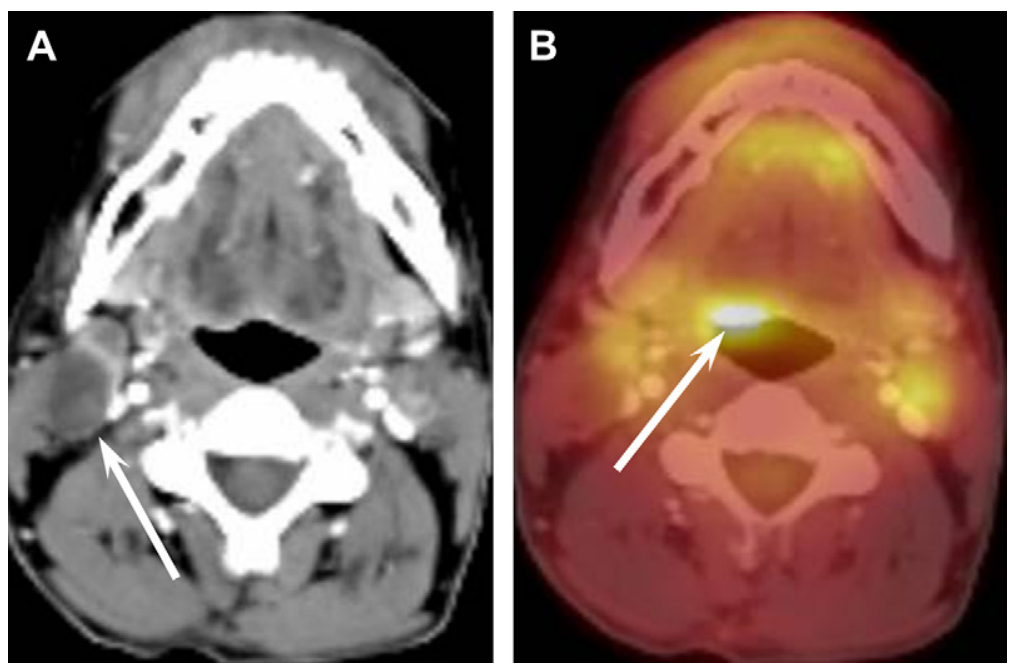

Fig. 4. Unknown primary tumor. A 43-year-old patient presented with an enlarging right neck mass. (A) CT shows a zone II neck mass (arrow), which FNA proved to be metastatic SCC. CT neck/chest, panendoscopy, and blind tongue base biopsy failed to disclose the primary tumor. (B) PET/CT examination identifies the patient's primary tumor (arrow) in the tongue base, confirmed with further biopsies. 
that the 10-year survival rate for HNSCC drops from $85 \%$ to $10 \%$ to $40 \%$ in patients with positive lymph nodes. One of the more controversial topics in the management of HNSCC is the clinically node-negative (N0) neck.

An N0 neck means that there is no clinical evidence of metatstatic disease within the neck. This may be based on physical exam of a neck with no palpable adenopathy or be based on a negative radiologic evaluation for cervical metastases. The majority of patients truly do not have metastatic disease within the cervical nodes. There is, however, an approximate $20 \%$ risk of occult metastases $[28,34]$ in patients with a clinically node-negative neck (Fig. 5).

Management of HNSCC with an N0 neck varies among institutions. Some clinicians advocate close observation while others choose to treat the nodes prophylactically, either with lymphadenectomy or external beam radiation. In patients in whom there is a low risk of occult metastases, observation is a reasonable recommendation. Proponents of observation feel that elective treatment of the neck in these patients would put patients through the risk of an unnecessary and potentially morbid procedure, which has not shown an increased survival benefit [35]. Given the risk of occult metastases, many surgeons however feel that an elective neck dissection is a relatively low morbidity procedure which can provide invaluable staging information, the benefits of which outweigh the risk of external beam radiation or having to deal with advanced neck disease after primary treatment.
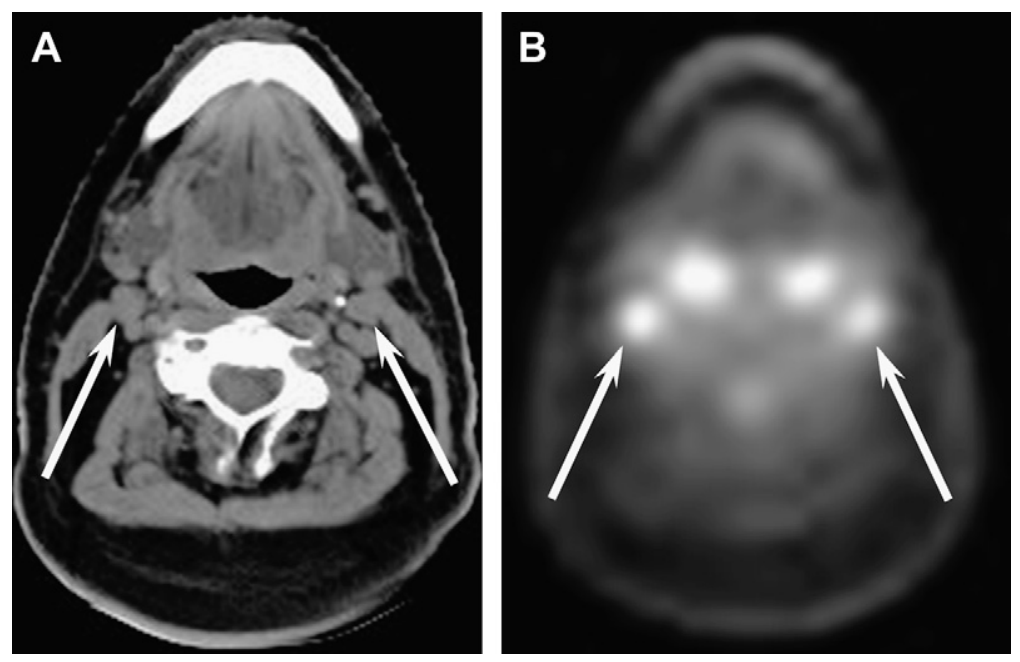

Fig. 5. The N0 neck. A 69-year-old male with 2-cm supraglottic SCC. Clinically, the patient had an N0 neck. (A) CT shows normal-sized, symmetric jugulodigastric nodes (arrows). (B) PET image at the same level demonstrates FDG avidity (arrows), which was confirmed as metastatic disease after neck dissection. 
Recent studies have focused on PET as a diagnostic tool for evaluating the presence of occult metastases. However, these studies have shown that PET is not accurate enough to preclude neck dissection and spare patients this additional procedure (Table 1) [36,37]. This is likely a reflection of the poor spatial resolution of PET imaging combined with its inability to detect nodal metastases less than $5 \mathrm{~mm}$ in diameter. PET/CT has not been studied extensively in the N0 neck but is presumed to perform similarly because of the inherent limitations of PET described previously. PET/CT cannot therefore preclude neck dissection in patients with advanced primaries but clinically node negative necks. Staging Squamous Cell Carcinoma key points below:

- $\mathrm{T} 1$ disease is unlikely to present with distant metastases, so radiologic staging is not necessary.

- T2 disease remains controversial.

- Whole-body PET/CT is the modality of choice for staging patients with advanced (T3 and T4) HNSCC.

- PET/CT finds $20 \%$ to $30 \%$ of unknown primary tumors above and beyond the traditional search, but PET/CT is a supplement, not a substitute, for endoscopy and biopsy.

- PET/CT is not sufficiently accurate to preclude neck dissection in patients with advanced primaries but clinically node negative (N0) necks.

\section{Treatment planning}

The current standard of care for patients with HNSCC involves combinations of surgery, radiation, and chemotherapy. Although the high doses of radiation used for treatment are effective, there is potential for significant toxicity, which leads to decreased quality of life and diminished patient compliance. Newer treatment modalities such as intensity-modulated radiation therapy (IMRT) and image-guided stereotactic radiosurgery (Gamma Knife and CyberKnife) aim to deliver radiation more efficiently and effectively. Essential to this is accurate delineation of targets. CT and MRI are traditionally used for target definition because of their high spatial resolution. These conventional imaging modalities are unfortunately insufficient for tumor definition in instances where the primary tumors are of small volume. Furthermore, as discussed earlier, CT/MRI criteria for lymph

Table 1

Articles evaluating the accuracy of PET/CT in evaluating the N0 neck

\begin{tabular}{lllc}
\hline Author and Year & No. Patients & Specificity, \% & Sensitivity, \% \\
\hline Wensing, et al, 2006 & 28 & 33 & 76 \\
Schoder, et al, 2006 & 36 & 67 & 85 \\
Stoekli, et al, 2002 & 12 & 25 & 88 \\
Myers, et al, 1998 & 12 & 78 & 100 \\
\hline
\end{tabular}


node involvement generally relies on size criteria and therefore diseased nodes may not be included in the radiation field if they are not "pathologically" enlarged.

There has been significant recent interest in the use of integrated PET/CT for radiotherapy planning. When compared with CT, PET/CT has been shown to more accurately assess gross target volume (GTV), which is essential to IMRT [38]. Numerous additional studies have showed similar results [39-43]. Because of its ability to provide both anatomic and functional information, PET/CT is able to provide for improved tumor coverage and sparing of normal tissues. This ultimately leads to improved treatment response while minimizing adverse effects. Treatment Planning summary below:

- PET/CT is superior to conventional imaging modalities for radiation treatment planning, allowing for improved tumor coverage and sparing of normal tissues.

\section{Monitoring treatment response}

Assessment of patients during and after therapy is vital to determine whether an alternate therapeutic approach is needed or whether additional therapy is warranted. Clinical examination is often insufficient to determine the effectiveness of treatment for HNSCC. Conventional cross-sectional imaging studies, while better than clinical examination, are still insensitive to subtle or early changes. Postsurgical and postradiation changes can complicate identification of recurrent or residual disease on physical exam and/or anatomic imaging with CT or MRI $[44,45]$. In addition, gadolinium enhancement on MRI can be seen in both residual tumor and treated, tumor-free soft tissues after chemoradiation.

PET alone, without combined CT, has been previously shown to be valuable in monitoring treatment response. It can detect metastatic disease and can be beneficial for differentiating radiation and surgical changes from residual or recurrent tumor because cancer cells retain more FDG for longer periods of time than normal tissues. Poor spatial resolution, however, makes anatomic localization difficult. Recent work has shown improvement using integrated PET/CT (Fig. 6). With sensitivity and specificity of $77 \%$ and $93 \%$, respectively, PET/CT had an overall accuracy of $86 \%$ for the detection of residual disease, and is superior to contrast-enhanced CT [46]. Similar studies showed PET/CT had sensitivity greater than $90 \%$ for localization of recurrent disease [47-49]. PET/CT is therefore a useful tool for monitoring treatment response in HNSCC and for restaging recurrences including nodal disease and distant metastases.

Management of patients with locoregionally advanced HNSCC (N2 or N3) has shifted from surgery and radiation to organ preservation therapy involving chemoradiation. Patients who do not show a complete response 

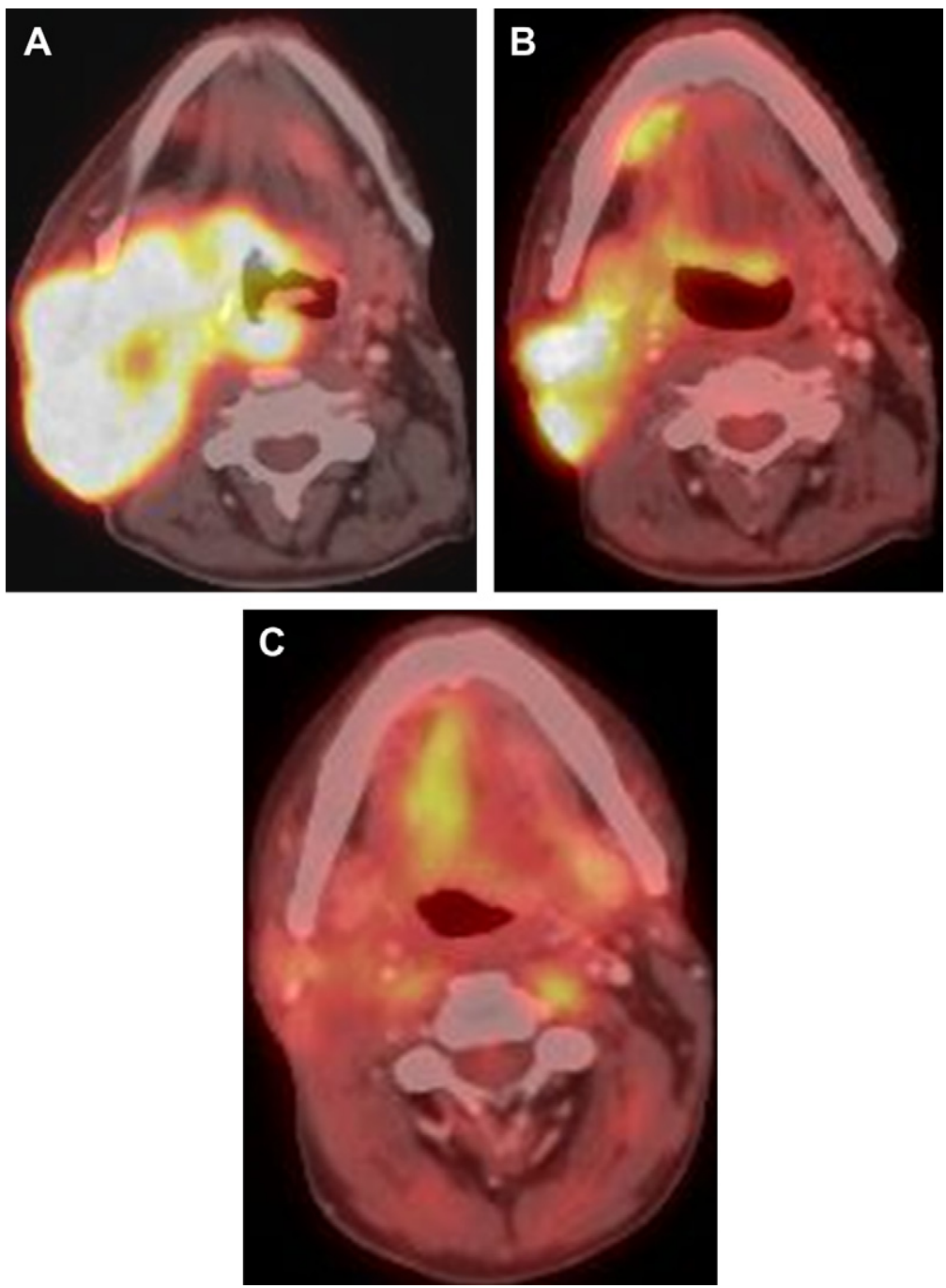

Fig. 6. Monitoring therapy response. (A) Staging PET/CT documents a massive HNSCC of the right neck. $(B)$ Two months after initiation of chemoradiation, dramatic improvement in tumor size and FDG avidity were noted, so therapy was continued. (C) Six months after initiation of chemoradiation, the tumor had resolved competely, with only physiologic uptake seen in the floor of mouth and paraspinal muscles.

after chemotherapy can then undergo a neck dissection. Those patients in whom there is a complete response may defer their neck dissection since isolated recurrence of disease within the neck is rare $(0 \%$ to $5 \%)$. This, however, is a controversial topic because of the lack of large-scale randomized trials evaluating neck dissection versus observation, especially in patients with a complete clinical response. 
Numerous studies have shown that PET/CT can detect residual or recurrent disease with more accuracy than conventional imaging, with specificities ranging from $92 \%$ to $97 \%$ [46-48,50,51]. In patients specifically with N2 or N3 nodal disease undergoing chemoradiation, surveillance imaging with PET/CT has been shown to have a very high negative predicted value (no false negatives), allowing confident exclusion of residual cancer thereby deferring a planned neck dissection (Fig. 7). Brkovich and colleagues [52] performed PET/CT at 8 to 12 weeks after chemoradiation treatment in 21 patients with various N-stage HNSCC and found a negative predicted value of $92 \%$. Likewise, Nayak and colleagues [53] performed PET/CT at 8 to 10 weeks after chemoradiation treatment in 30 patients with $\mathrm{N} 2$ or N3 HNSCC and found a negative predicted value of $100 \%$. PET/CT therefore has the potential to prevent unnecessary neck dissections in patients with nodal disease that responds completely to nonsurgical modalities. Monitoring Treatment Response summary below:

- PET/CT is useful for monitoring treatment response in HNSCC.

- PET/CT is useful for restaging, including nodal disease and distant metastases.

- PET/CT has the potential to prevent unnecessary neck dissections in patients with nodal disease that responds completely to therapy.
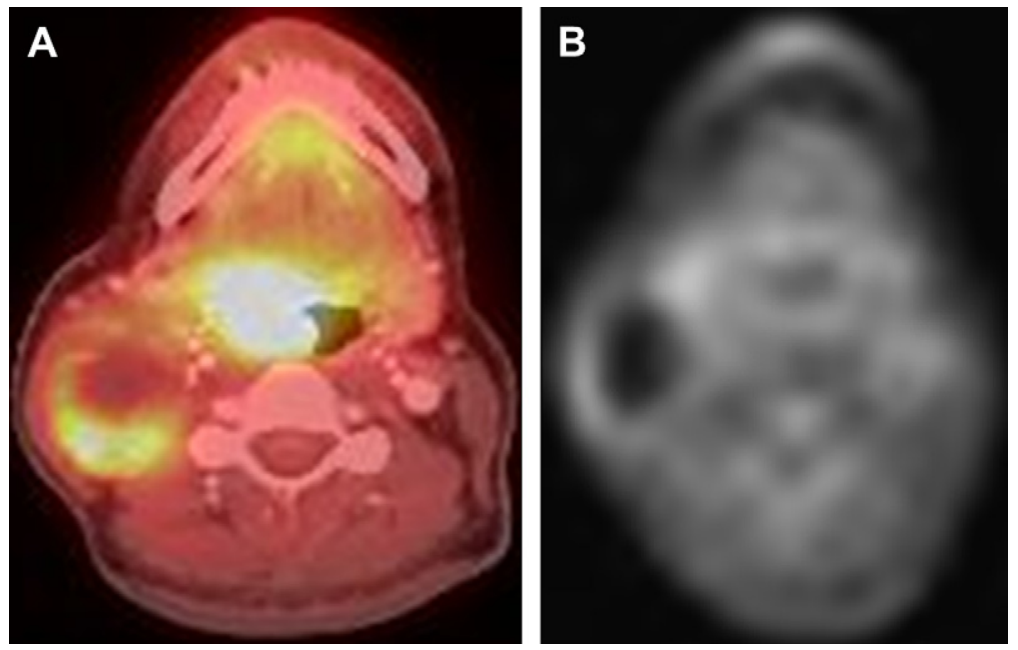

Fig. 7. Deferring planned neck dissection. A 56-year-old male with T4 N2a SCC of right tongue base. (A) Staging PET/CT exam shows extensive primary and large necrotic metastasis. $(B)$ PET/CT after completion of chemoradiation shows no residual abnormal FDG avidity in the neck, despite the persistence of a palpable mass. Neck dissection was deferred, and the patient is without recurrence after 2 years. 


\section{Surveillance}

Given recurrence rates as high as $50 \%$ for patients with advanced-stage SCC, posttreatment radiologic surveillance allows for early and accurate detection of recurrent disease. This leads to improved survival with surgery or re-irradiation [51-53]. Patients with primary HNSCC are more likely to develop second primary cancers than any other group of cancer patients.

\section{Surveillance protocol}

PET and PET/CT have proven to be the modalities of choice for surveillance of patients with HNSCC. However, some debate still exists over the optimal timing of the first surveillance scan.

Early scanning with PET/CT has low sensitivity because there may be few viable tumor cells and those cells may be stunned from therapy. Additionally, uptake in the head and neck on PET/CT in the early post-treatment period may reflect post-operative or post-radiotherapy inflammation. In one study of patients with HNSCC treated with primary radiotherapy, all inaccurate treatment follow-up PET/CT readings occurred between 4 and 8 weeks after therapy [46]. Similarly, Ryan and colleagues [51] showed that the sensitivity of PET rose dramatically 1 month after therapy from $55 \%$ to $95 \%$, as did the negative predicted value from $90 \%$ to $99 \%$. Initial surveillance with PET/CT should therefore be performed at least two months after the conclusion of therapy to decrease the number of false positives (Fig. 8).
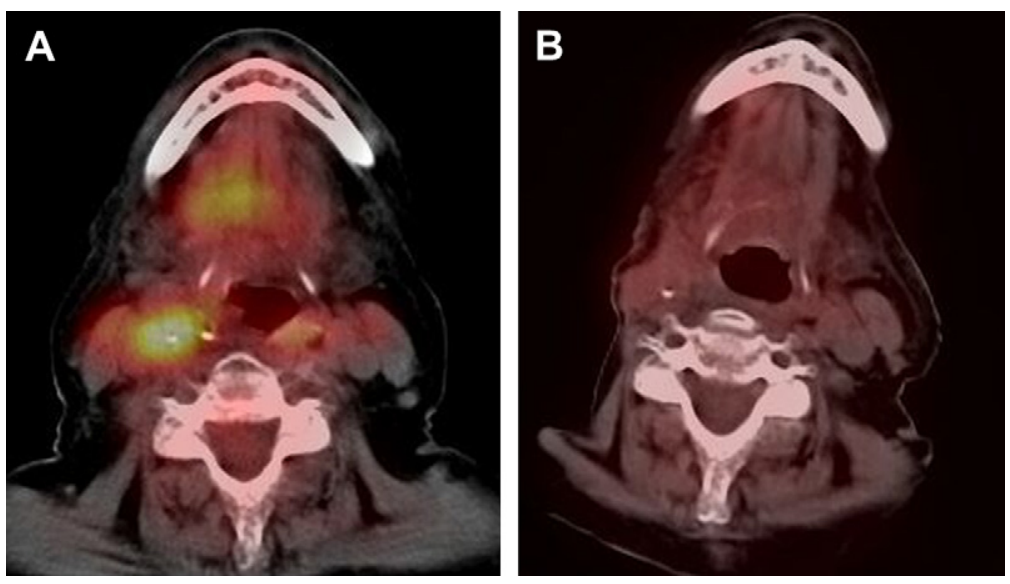

Fig. 8. False-positive PET/CT performed too soon after therapy. (A) PET/CT performed 4 weeks after conclusion of chemoradiation shows concerning residual FDG uptake in right Zone 2 nodes. $(B)$ Four weeks later, this uptake has resolved despite no interval therapy. Initial surveillance PET/CT should be performed a minimum of 8 weeks after the conclusion of therapy. 
Short-interval follow-up examinations with PET/CT can detect recurrence in patients who have completed or are undergoing treatment for HNSCC $[47,48,50,51,54]$. To date, there have been no published studies to determine the optimal surveillance interval for these patients. At our institution, patients with HNSCC are scanned with PET/CT at 2 months, 5 months, 8 months, and 14 months after treatment. Patients with scans suggesting recurrence undergo tissue sampling (either open or CT-guided biopsy), while patients with questionably abnormal findings undergo additional PET/CT 3 months later. Clinical follow-up is performed indefinitely in these patients, even after the PET/CT portion of the regimen is concluded. Worrisome clinical findings at any point may prompt an additional PET/CT scan. Patients who have undergone multimodality therapy can be especially difficult to assess clinically; in this situation, PET/CT is particularly helpful. Analysis of this surveillance regimen is pending, and similar trials are ongoing at other institutions.

Patients with HNSCC should be closely followed both clinically and radiographically within the first year after treatment given that most recurrences present within that first year after therapy. But it remains unclear how long a patient should be followed radiographically after being declared disease free [55]. To date, there have been no published studies that address this question. Given that most cancers will recur within 1 year, the authors choose to end radiographic surveillance after 14 months of negative PET/ CT scans. There will, however, inevitably be some patients whose cancer recurs after concluding radiographic surveillance (Fig. 9). Any patient who presents with clinical evidence of recurrent disease merits PET/CT scanning, regardless of the time since therapy.
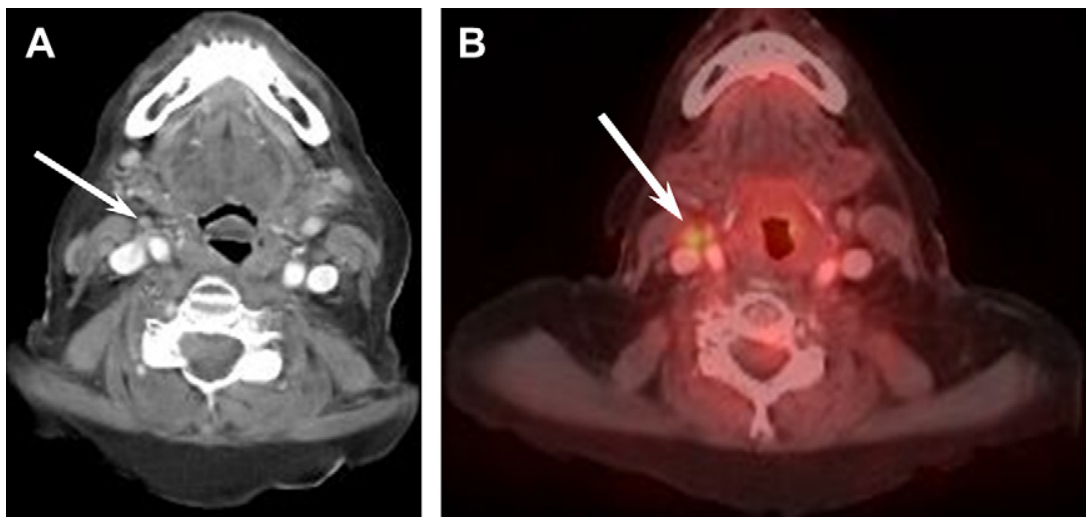

Fig. 9. Late recurrence. A 60-year-old woman with T3 N1 SCC of hypopharynx treated successfully with chemoradiation (negative PET/CTs for 2 years). (A) PET/CT performed 19 months after therapy showed no active disease. The lymph node marked with an arrow is normal by radiographic criteria, but PET/CT at 26 months $(B)$ demonstrates a recurrence in that node (arrow). Any reasonable surveillance regimen should expect a few patients to recur outside the surviellance window. 


\section{Second primary}

Patients treated for primary HNSCC have a high rate $(3 \%$ to $5 \%$ per year) of developing second primary tumors [56]. This most likely is because of the widespread distribution of toxic effects from tobacco and alcohol, the two most important risk factors for HNSCC. Second cancers can occur around the same time as the primary (synchronous) or 6 months or more after the primary (metachronous). The major sites of involvement are the head and neck, lung, and esophagus.

Posttreatment surveillance can diagnose these second primary lesions earlier and more accurately allowing for potentially higher cure rates. Panendoscopy at regular intervals following treatment has not been shown to be effective in detecting second primary tumors, and, as a result, treatment guidelines issued by the American Head and Neck Society do not recommend routine posttreatment panendoscopy. Some clinicians have suggested that panendoscopy only be used when patients present with new onset of worrisome symptoms (ie, dysphagia, odynophagia, hoarseness, and so forth).

PET and PET/CT have proven useful for detection of second primary tumors in the head and neck and elsewhere in the body in patients with previously treated HNSCC being evaluated for recurrence (Fig. 10). Perlow and colleagues [54] found 10 second primary lung tumors in 41 patients being evaluated for HNSCC recurrence with PET. In fact, PET has been shown to detect higher rates of synchronous lung lesions than standard chest $\mathrm{CT}$ alone [22]. PET/CT is therefore the modality of choice for identifying second primary tumors. Although the authors do not advocate surveillance for this indication alone, it is an important added benefit for patients undergoing post-therapy surveillance or restaging. Surveillance Scanning summary below:

- PET/CT is highly accurate for identifying recurrent SCC.

- Initial surveillance PET/CT should be performed at least 2 months after conclusion of therapy.

- No definitive conclusions can yet be drawn regarding optimal surveillance interval or appropriate length of surveillance in patients with HNSCC.

- Surveillance with PET/CT allows better detection of second primary tumors.

\section{Thyroid carcinoma}

According to the American Cancer Society, approximately 30,000 new cases of thyroid cancer are diagnosed in the Unites States each year. Two thirds of cases will be diagnosed in women and two thirds of patients will be between the ages of 20 and 55. Although thyroid cancer carries one of the best 5-year survival outcomes among cancers (97\%), there are still roughly 1500 deaths each year [1]. 

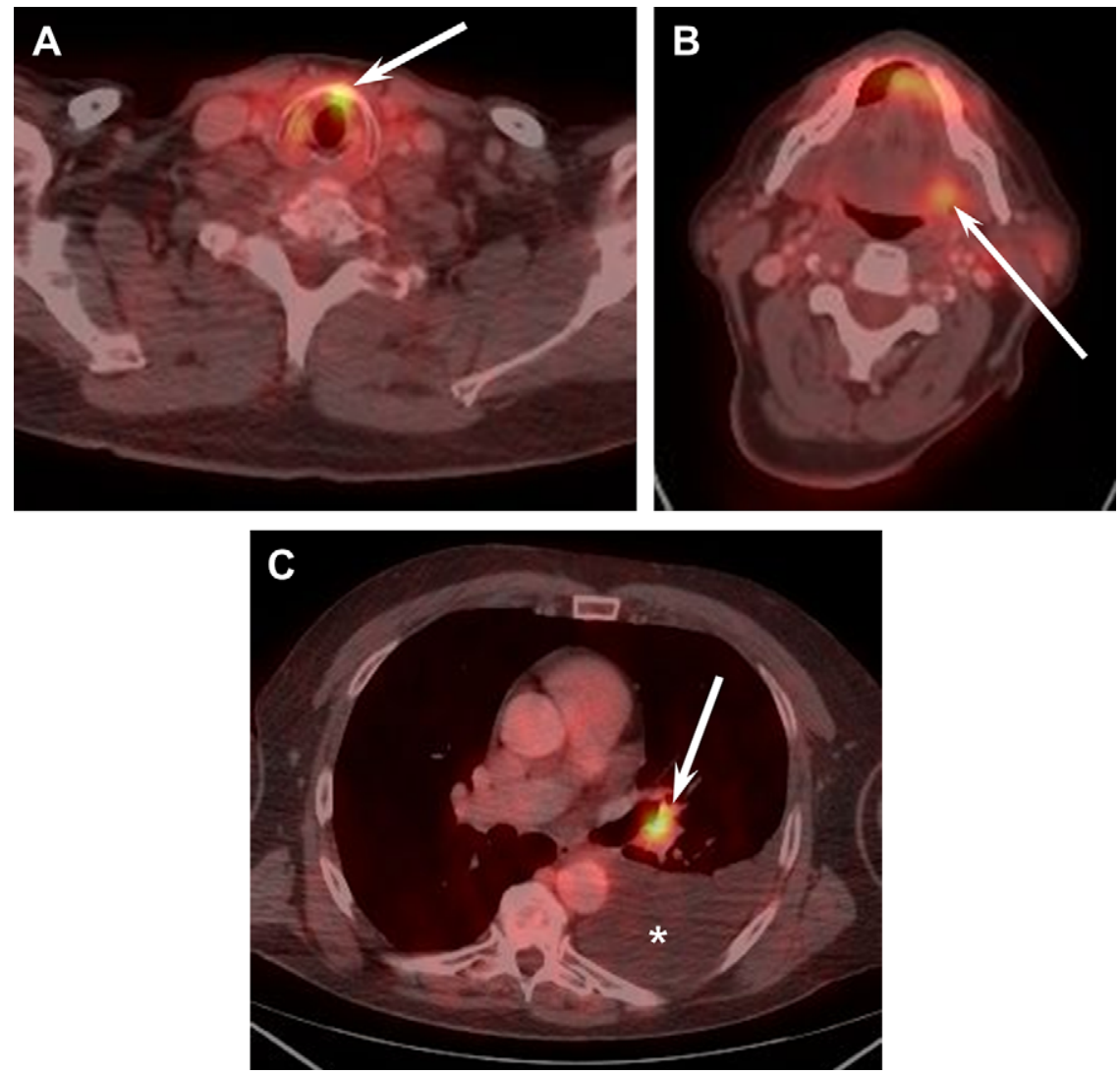

Fig. 10. Second primary tumors. A 63-year-old man with recurrent glottic cancer. $(A)$ The laryngeal recurrence (arrow) is well-documented with PET/CT. (B) An unexpected focus of FDG uptake (arrow) is noted in the left retromolar trigone, which proved to be a second primary tumor. (C) A focus of FDG uptake (arrow) is seen in the central left lung, accompanied by a pleural effusion (*), representing yet another unexpected primary tumor. PET/CT is useful for detecting second primary tumors in patients undergoing restaging or surveillance.

Tumors within the thyroid gland can be either benign $(95 \%)$ or malignant $(5 \%)$. Malignant thyroid tumors can be grouped by their cell of origin with papillary and follicular carcinomas collectively classified as differentiated (DTC) because they maintain their ability to trap iodine and produce thyroglobulin. Medullary carcinoma develops from C cells that make calcitonin, and anaplastic carcinoma is the undifferentiated form of thyroid cancer.

\section{Differentiated thyroid carcinoma}

The current standard of treatment for DTC is total thyroidectomy followed by treatment with I-131 for microscopic residual disease. PET has not been shown to be of benefit in the preoperative staging of patients diagnosed with DTC because the physiologic uptake of the gland will 
prevent assessment of the primary tumor. In addition, false positives may be seen because of the uptake in brown fat and muscles of speech.

Recurrences of DTC tend to occur in the first 5 years after initial treatment. Identifying recurrent/residual disease allows for more effective early treatment either with repeat surgery or external beam radiation. Patients are monitored for recurrence using serum thyroglobulin levels, which is a sensitive marker for residual/recurrent disease and is used in conjunction with whole body I-131 scans for localization of disease [57]. Whole body nuclear medicine scans may, however, be negative in up to $15 \%$ of patients with detectable thyroglobulin levels. Tumor volume may be below the threshold for detection by scintigraphy or tumor cells may have lost their ability to trap iodine but retained their ability to secrete thyroglobulin.

PET has been shown to be of value in evaluating patients for disease recurrence when the patients were treated with total thyroidectomy and radioiodine, present with increased serum thyroglobulin, and have a negative whole body I-131 scan [58]. Definitive anatomic localization is, however, needed to direct surgical management. Recent studies have shown that integrated PET/CT has improved diagnostic accuracy over PET alone and over side-by-side PET and CT imaging (Fig. 11). PET/CT can differentiate physiologic from pathologic FDG uptake. Providing both anatomic and metabolic information, PET/CT has an increased diagnostic accuracy $(93 \%)$ compared with PET alone (78\%) for precise localization of tumor tissue and can change management in a significant percentage of patients [59]. PET/CT should therefore be used in the evaluation of patients with treated DTC who present with elevated thyroglobulin levels and a negative radioiodine scan.

\section{Medullary thyroid carcinoma}

Medullary thyroid carcinoma (MTC) arises from C cells in the thyroid gland. It is distinguished from DTC because of its inability to trap iodine and its production of calcitonin. MTC spreads to lymph nodes in the neck and mediastinum and later disseminates to the lungs, bones, and liver. It is relatively radioresistant and therefore an aggressive surgical approach with total thyroidectomy coupled with aggressive nodal resection is the treatment of choice.

Serum calcitonin levels are extremely sensitive and specific for residual or recurrent disease and are monitored postoperatively. Calcitonin-positive patients require further evaluation for anatomic localization, usually with sonography. Various other nuclear medicine tests have also been used with limited efficacy. CT and MRI have been shown to have higher tumor detection rates than ultrasound, but small lesions still may be missed. PET is more sensitive than other imaging modalities for detection of residual or recurrent MTC [60], but unfortunately not all MTC is FDG-avid [4]. 

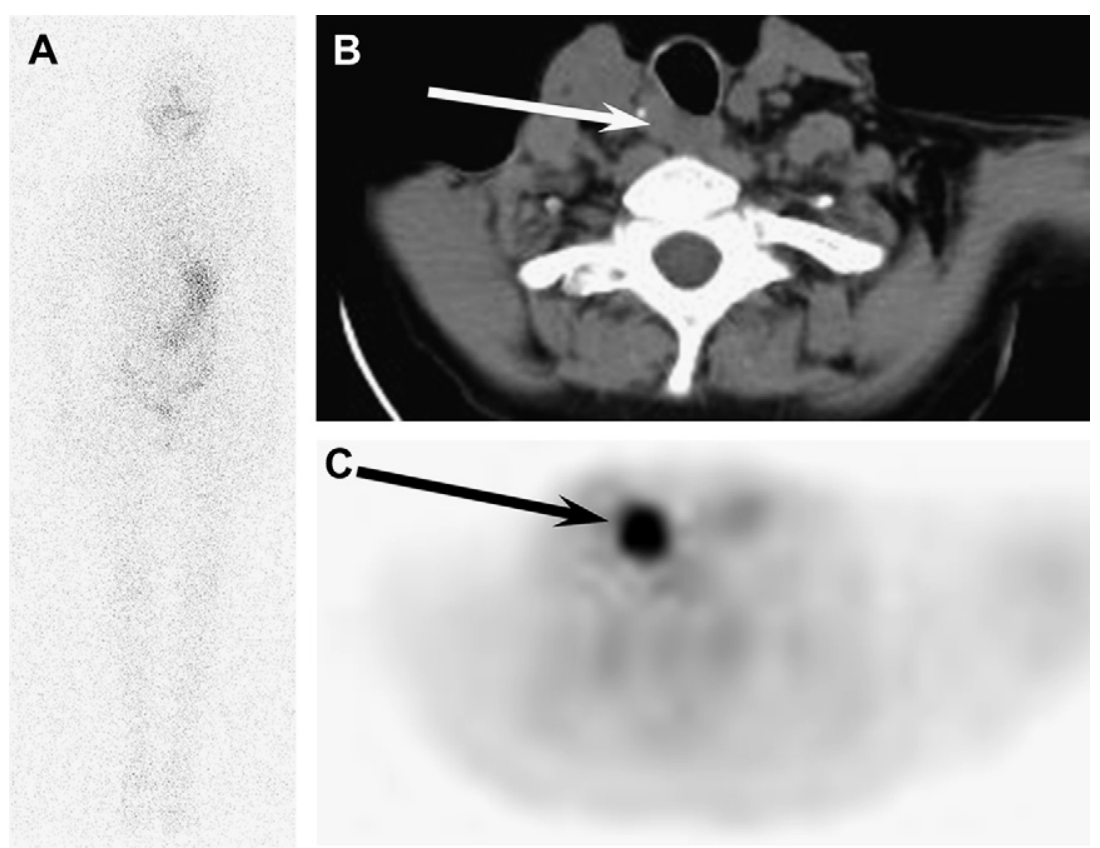

Fig. 11. Differentiated thyroid cancer. A 28-year-old woman with papillary thyroid carcinoma, tall cell variant, underwent thyroidectomy and central compartment dissection, followed by radioiodine ablation therapy, and now presents with elevated thyroglobulin levels. $(A)$ Wholebody I-131 scan does not show any areas of abnormal radiotracer activity to suggest residual or metastatic disease. CT $(B)$ and PET $(C)$ show a focal area of increased FDG activity corresponding to abnormal tissue (arrows) within the tracheo-esophageal groove, which proved to be recurrent thyroid carcinoma.

Also, PET provides only limited morphological information for precise anatomic localization at surgery (Fig. 12). PET/CT can fill this void by providing exact anatomic direction to the surgeon based on areas of increased uptake [61]. PET/CT can thus be recommended in patients with persistent measurable calcitonin levels after total thyroidectomy and equivocal or negative sonography, but a lack of FDG uptake should not necessarily be interpreted as absence of disease.

\section{Anaplastic thyroid carcinoma}

Anaplastic thyroid carcinoma is a rare but highly malignant tumor composed of undifferentiated malignant cells. The rapid and aggressive growth of the tumor means survival for most patients is measured in months. Patients with potentially curable disease should be treated with surgery; the use of radiation and chemotherapy is controversial.

Diagnostic imaging with ultrasound, CT, and/or MRI is useful for defining the extent of disease and treatment planning. Evaluation for distant metastases is guided by clinical and laboratory findings and usually 

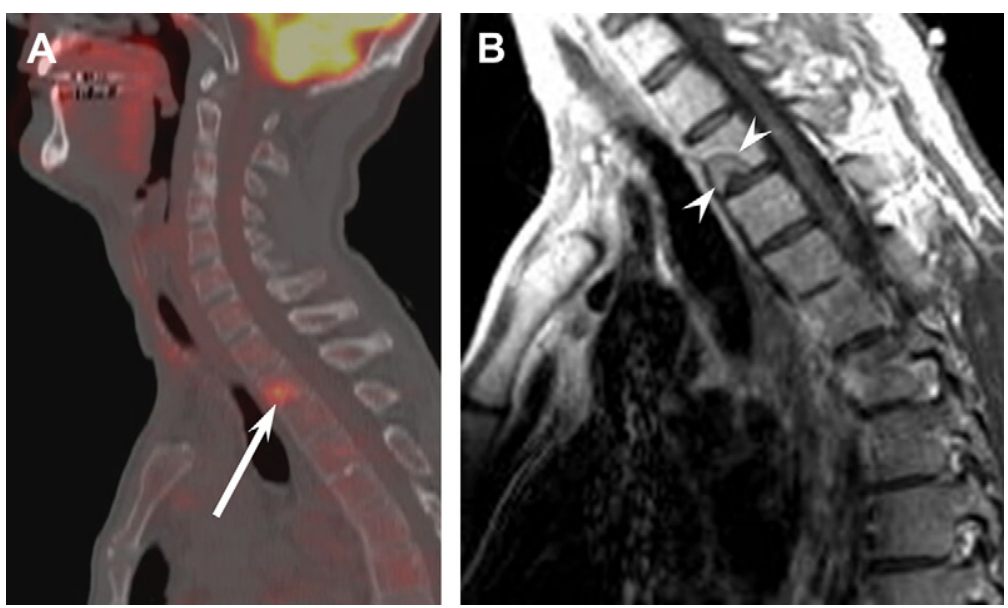

Fig. 12. Metastatic medullary thyroid carcinoma. (A) Sagittal PET/CT shows a focus of increased uptake (arrow) in an upper thoracic vertebral body that might be confused with spondylitis. (B) Sagittal T1-weighted MR confirms the presence of a mass (arrowheads) that proved to be metastatic medullary thyroid carcinoma.

performed with chest CT, radiographs, and/or whole body bone scan. Although there is limited research regarding the role of PET and PET/CT in anaplastic thyroid carcinoma, initial reports suggest that it can be used to help define the local extent of disease and determine the presence or absence of distant metastases (Fig. 13) [58]. Because therapy is generally not affected by the results of PET/CT, however, the cost is usually not warranted. Thyroid Cancer summary below:

- PET/CT should be used in the evaluation of patients with treated differentiated thyroid carcinoma who present with elevated thyroglobulin levels and a negative radioiodine scan.

- PET/CT should be considered in the evaluation of patients with treated medullary thyroid carcinoma who present with persistent measurable calcitonin levels and equivocal or negative sonography.

- PET/CT may help to define local extent of disease and presence of distant metastases in patients with anaplastic thyroid carcinoma, but the cost is not generally warranted, as the prognosis is fixed.

\section{Lymphoma and melanoma}

The incidence of Hodgkin's disease (HD) and non-Hodgkin's lymphoma (NHL) have been rising 3\% to $5 \%$ per year [1]. Because these diseases are potentially curable, accurate staging and effective treatment monitoring are essential. CT has been the traditional method for staging and monitoring lymphoma. However, CT relies mainly on anatomic criteria, most notably size, and may underestimate disease. PET has been shown to be extremely 


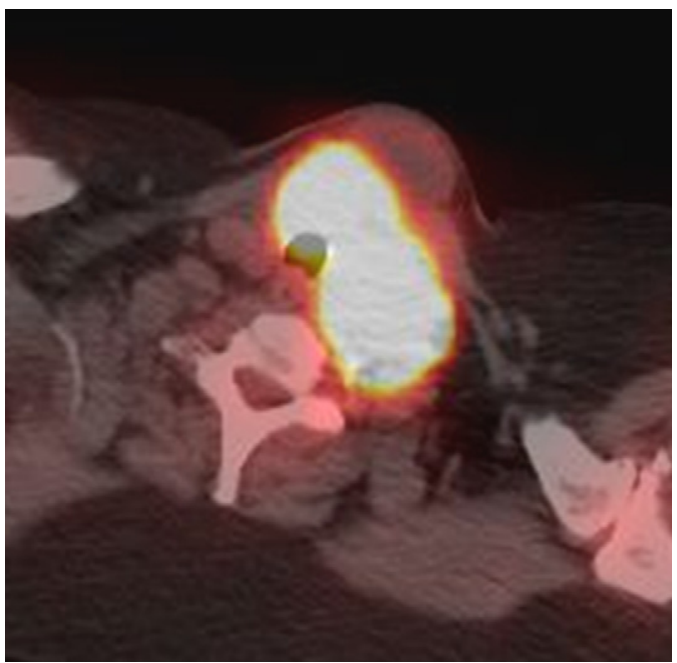

Fig. 13. Anaplastic thyroid carcinoma. PET/CT shows marked FDG avidity of the tumor, but the results of this test are unlikely to change therapy or affect prognosis.

accurate for detection of disease but is limited by poor anatomic landmarks. PET/CT overcomes these shortcomings by combing both anatomic and physiologic information (Fig. 14). With more exact anatomic localization, $\mathrm{PET} / \mathrm{CT}$ results in more accurate staging and restaging of patients with lymphoma, both in the head and neck and elsewhere in the body [62].

Melanoma is an aggressive neoplasm of the skin and other mucosal surfaces that can spread in an unpredictable manner and involve any site in the body through direct extension, lymphatic invasion, or hematogenous spread. Imaging is able to detect unsuspected lesions in settings where the initial lesion may be clinically apparent. CT is currently the most common imaging study used for evaluation of patients with melanoma, but melanoma consistently shows dramatic FDG uptake on PET studies (Fig. 15). Thus, PET/CT is playing an increasing role in the management of these patients, and has improved diagnostic accuracy for both detection of disease and treatment monitoring when compared with both conventional imaging modalities and PET alone [63-65]. Lymphoma and Melanoma summary below:

- PET/CT is indicated for staging, assessing response to therapy, and surveillance in patients with lymphoma and melanoma.

\section{Recommendations for success}

Standardized uptake values

Clinical and pathological factors such as tumor stage and histological subtype have been used to predict outcome in patients with HNSCC. 

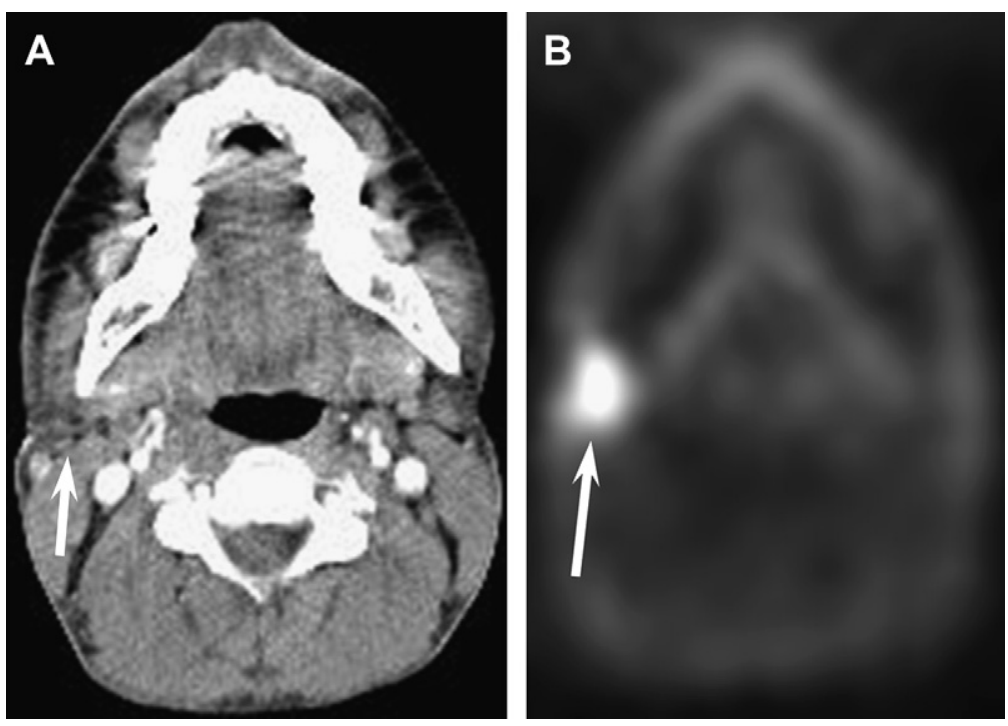

Fig. 14. Head and neck lymphoma. A 43-year-old woman with a palpable mass at her right parotid tail. (A) CT reveals no mass in the area of interest (arrow). (B) PET image at the same level shows marked uptake in the parotid tail (arrow), which later proved to be parotid lymphoma. PET/CT is extremely sensitive for the presence of lymphoma.

With increasing use of PET and PET/CT, there has been significant interest in the use of the standardized uptake value (SUV) to aid in prognosis. SUV is a semiquantitative measurement of tumor uptake of FDG derived by measuring the ratio of radioactivity in the tumor to the expected baseline activity in the body. Several studies have shown the potential for SUV to predict outcome in patients with HNSCC [66-68]. There are, however, several limitations regarding the use of SUV. No consensus method exists for the calculation of SUV to allow for standardization. In addition, there are numerous variables (both technical and patient-related) that can affect the amount of tracer uptake within tissues thereby causing variations in SUV from patient to patient and scan to scan $[69,70]$. Threshold values for categorizing lesions as benign or malignant using SUV have not been adequately verified, and most head and neck radiologists now eschew strict reliance on SUV. Further research is needed to determine whether SUV can be incorporated with other factors to provide accurate prognoses.

\section{Fusion techniques}

There are four methods of performing PET/CT:

(1) Acquisition of PET and CT images on separate scanners with post hoc fusion

(2) Combined scanning with low-dose CT for attenuation correction only 


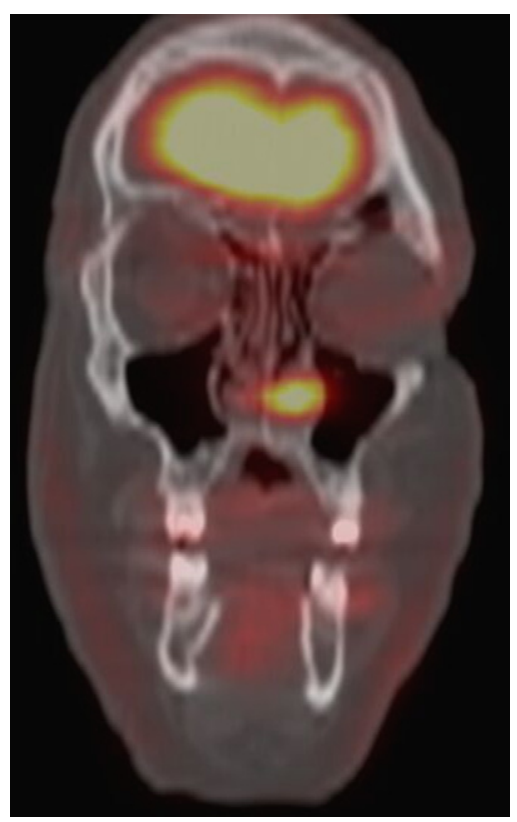

Fig. 15. Nasal melanoma. Coronal PET/CT image shows marked uptake in a left nasal cavity mass, which proved to be a primary nasal melanoma. Like lymphoma, melanoma is consistently FDG-avid, and PET/CT is very useful to assess for metastatic disease.

(3) Combined scanning with unenhanced CT for anatomic correlation

(4) Combined scanning with diagnostic quality-enhanced CT

Misregistration artifacts due to repositioning of the patient for a second scan make the post hoc fusion suboptimal for evaluation of patients with HNSCC. Both low-dose CT and unenhanced CT may not provide the detail required to accurately assess the head and neck for subtle disease pathology. Although the use of intravenous (IV) contrast may create additional attenuation-correction artifacts, radiologists who are familiar with these artifacts will not lose clinical accuracy [4]. Some of the prior literature showing suboptimal results from PET/CT in head and neck cancer may have relied on nondiagnostic CT scans, and thus not be comparable with more recent reports. Readers should be attentive to the type of scans performed when interpreting results from the literature.

\section{Interpretation}

In most clinical settings, a single radiologist interprets combined PET/CT examinations [71]. The fusion of two separate imaging modalities, however, creates a unique set of artifacts on both the CT and PET portions of the examination. A radiologist with experience in both head and neck imaging and PET imaging is therefore needed for optimal interpretation. In fact, 
dual readings by radiologists with expertise in nuclear medicine and neuroradiology may be necessary until interpretations can be made comfortably independently.

\section{Clinically relevant reporting}

Because PET/CT combines information from two sources, it is easy for radiologists to end up with equivocal findings, especially if the two modalities produce discrepant results. It is important for the interpreting radiologist to have a full understanding of the clinical context in which the examination was performed. This requires close communication with the referring physician. Optimal radiology reports will contain recommendations for further testing, when appropriate, to improve the level of certainty in the diagnosis.

At our institution, radiology reports are strictly classified into one of four categories:

(1) No evidence of malignancy

(2) Abnormality unlikely to represent tumor-follow radiographically in 3 months

(3) Suspicious for malignancy-direct inspection or tissue sampling warranted

(4) Definite malignancy

With this scheme in place, referring physicians can efficiently plan further care upon reading the radiology report. Of course, clinical parameters and physical examination are still the primary guides for therapeutic decisions, but with a reproducible and predictable radiology reporting scheme, the radiologic information can be given appropriate weight.

\section{Multidisciplinary care}

A multidisciplinary team approach is essential for effective treatment planning in head and neck cancer. Consensus review of patient data by medical oncology, radiation oncology, otolaryngology, and radiology not only allows for clear definition of treatment strategies and goals but also ensures patients receive appropriate follow-up care. Radiologic data can be presented in a clinically relevant context that minimizes the confusion and imprecision inherent in any written radiology report. Recommendations for Success with PET/CT summary below:

- Beware of strict SUV thresholds when assessing PET/CT. An experienced radiologist's subjective opinion will be more accurate.

- A combined PET/CT scanner, using a diagnostic-quality contrastenhanced CT protocol, will produce optimal results.

- A radiologist with experience in both head and neck imaging and PET imaging is important for accurate interpretation of head and neck $\mathrm{PET} / \mathrm{CT}$. 
- Reproducible, clinically relevant reporting schemes will minimize uncertainty and decrease equivocal results in radiology reports.

- A multidisciplinary team conference provides the best environment for communication of clinically relevant radiologic data.

\section{References}

[1] American Cancer Society. Available at: (www.cancer.org). Accessed October 28, 2007.

[2] Forastiere A, Koch W, Trotti A, et al. Head and neck cancer. N Engl J Med 2001;345: 1890-900.

[3] Minn H, Paul R, Ahonen A. Evaluation of treatment response to radiotherapy in head and neck cancer with fluorine-18 fluorodeoxyglucose. J Nucl Med 1998;29(9):1521-5.

[4] Branstetter BF, Blodgett TM, Zimmer LA, et al. Head and neck malignancy: Is PET/CT more accurate than PET or CT alone? Radiology 2005;235(2):580-6.

[5] Schoder H, Yeung HWD, Gonen M, et al. Head and neck cancer: clinical usefulness and accuracy of PET/CT image fusion. Radiology 2004;231:65-72.

[6] Bar-Shalom R, Yefremov N, Guralnik L, et al. Clinical performance of PET/CT in evaluation of cancer: additional value for diagnostic imaging and patient management. J Nucl Med 2003;44(8):1200-9.

[7] Zanation AM, Sutton DK, Couch ME, et al. Use, accuracy and implications for patient management of [18F]-2-fluorodeoxyglucose-positron emission/computerized tomography for head and neck tumors. Laryngoscope 2005;115(7):1186-90.

[8] Blodgett TM, Fukui MB, Snyderman CH, et al. Combined PET-CT in the head and neck part 1. Radiographics 2005;25(4):897-912.

[9] Greene FL, Page DL, Fleming ID, et al. AJCC Cancer Staging Manual. 6th edition. New York: Springer-Verlag; 2002.

[10] Prehn RB, Pasic TR, Harari PM, et al. Influence of computed tomography on pretherapeutic tumor staging in head and neck cancer patients. Otolaryngol Head Neck Surg 1998;119(6): $628-33$.

[11] Laubenbacher C, Saumweber D, Wagner-Manslau C, et al. Comparison of fluorine-18-fluorodeoxyglucose PET, MRI and endoscopy for staging head and neck sqamous-cell carcinomas. J Nucl Med 1995;36(10):1747-57.

[12] Wong WL, Chevretton E, McGurk M, et al. A prospective study of PET-FDG imaging for the assessment of head and neck squamous cell carcinoma. Clin Otolaryngol 1997;22(3): 209-14.

[13] Shah NP, Workman RB, Coleman RE, et al. "PET and PET/CT in head and neck cancer". In: Workman RB, Coleman RE, editors. PET/CT essentials for clinical practice. New York: Springer; 2006. p. 104-29.

[14] Hannah A, Scott AM, Tochon-Danguy H, et al. Evaluation of 18 F-fluorodeoxyglucose positron emission tomography and computed tomography with histopathologic correlation in the initial staging of head and neck cancer. Ann Surg 2002;236:208-17.

[15] Schmid DT, Stoeckli SJ, Bandhauer F, et al. Impact of positron emission tomography on the initial staging and therapy in locoregional advanced squamous cell carcinoma of the head and neck. Laryngoscope 2003;113:888-91.

[16] Don DM, Anzai Y, Lufkin RB, et al. Evaluation of cervical lymph node metastases in squamous cell carcinoma of the head and neck. Laryngoscope 1995;105(7):669-74.

[17] King AD, Tse GMK, Ahuja AT, et al. Necrosis in metastatic neck nodes: diagnostic accuracy of CT, MR imaging and US. Radiology 2004;230(3):720-6.

[18] Adams S, Baum RP, Stuckensen T, et al. Prospective comparison of 18F-FDG-PET with conventional imaging modalities (CT, MRI, US) in lymph node staging of head and neck cancer. Eur J Nucl Med 1998;25(9):1255-60. 
[19] Schwartz DL, Ford E, Rajendran J, et al. FDG-PET/CT imaging for preradiotherapy staging of head-and-neck squamous cell carcinoma. Int J Radiat Oncol Biol Phys 2005;61: $129-36$.

[20] Koshy M, Paulino AC, Howell R, et al. F-18 FDG PET-CT fusion in radiotherapy treatment planning for head and neck cancer. Head Neck 2005;27(6):494-502.

[21] Ha PK, Hdeib A, Goldenberg D, et al. The role of positron emission tomography and computed tomography fusion in the management of early-stage and advanced-stage primary head and neck squamous cell carcinoma. Arch Otolaryngol Head Neck Surg 2006;132(1): $12-6$.

[22] Goerres GW, Schmid DT, Gratz KW, et al. Impact of whole body positron emission tomography on initial staging and therapy in patients with squamous cell carcinoma of the oral cavity. Oral Oncol 2003;39(6):547-51.

[23] Brouwer J, de Bree R, Hoekstra OS, et al. Screening for distant metastases in patients with head and neck cancer: is chest computed tomography sufficient? Laryngoscope 2005;115(10): $1813-7$.

[24] Reiner B, Siegel E, Sawyer R, et al. The impact of routine CT of the chest on the diagnosis and management of newly diagnosed squamous cell carcinoma of the head and neck. Am J Roentgenol 1997;169(3):667-71.

[25] de Bree R, Deurloo EE, Snow GB, et al. Screening for distant metastases in patients with head and neck cancer. Laryngoscope 2000;110(3 Pt 1):397-401.

[26] Troell RJ, Terris DJ. Detection of metastases from head and neck cancers. Laryngoscope 1995;105(3):247-50.

[27] Teknos TN, Rosenthal EL, Lee D, et al. Positron emission tomography in the evaluation of stage III and IV head and neck cancer. Head Neck 2001;23(12):1056-60.

[28] Quon A, Fischbein NJ, McDougall IR, et al. Clinical role of 18F-FDG PET/CT in the management of squamous cell carcinoma of the head and neck and thyroid carcinoma. J Nucl Med 2007;48(1):58S-67S.

[29] Johansen J, Eigtved A, Buchwald C, et al. Implication of 18F-fluoro-2-deoxy-D-glcose positron emission tomography on management of carcinoma of unknown primary in the head and neck: a Danish cohort study. Laryngoscope 2002;112(11):2009-14.

[30] Jungehulsing M, Scheidhauer K, Damm M, et al. 2(F)-fluoro-2-deoxy-D-glucose positron emission tomography is a sensitive tool for the detection of occult primary cancer (carcinoma of unknown primary syndrome) with head and neck lymph node manifestation. Otolaryngol Head Neck Surg 2000;123(3):294-301.

[31] Miller FR, Hussey D, Beeram M, et al. Positron emission tomography in the management of unknown primary head and neck carcinoma. Arch Otolaryngol Head Neck Surg 2005; 131(7):626-9.

[32] Rusthoven KE, Koshy M, Paulino AC, et al. The role of fluorodeoxyglucose positron emission tomography in cervical lymph node metastases from an unknown primary tumor. Cancer 2004;101(11):2641-9.

[33] Gutzeit A, Antoch G, Kuhl H, et al. Unknown primary tumors: detection with dual-modality PET/CT—initial experience. Radiology 2005;234(1):227-34.

[34] Schoder H, Carlson DL, Kraus DH, et al. 18f-FDG PET/CT for detecting nodal metastases in patients with oral cancer staged N0 by clinical examination and CT/MRI. J Nucl Med 2006;47:755-62.

[35] Layland MK, Sessions DG, Lenox J, et al. The influence of lymph node metastasis in the treatment of squamous cell carcinoma of the oral cavity, orophayrnx, larynx and hypopharynx: N0 versus N+. Larngoscope 2005;115(4):629-39.

[36] Stoekli SJ, Steinert H, Pfaltz M, et al. Is there a role for positron emission tomography with $18 \mathrm{~F}$-fluorodeoxyglucose in the initial staging of nodal negative oral and oropharyngeal squamous cell carcinoma. Head Neck 2002;24:345-9.

[37] Myers LL, Wax MK, Nabi H, et al. Positron Emission Tomography in the Evaluation of the N0 Neck. Laryngoscope 1998;108(2):232-6. 
[38] Wang D, Schultz CJ, Jursinic PA, et al. Initial experience of FDG-PET/CT guided IMRT of head and neck carcinoma. Int J Radiat Oncol Biol Phys 2006;65(1):143-51.

[39] Heron DE, Andrade RS, Flickinger J, et al. Hybrid PET-CT simulation for radiation treatment planning in head-and-neck cancers. A brief technical report. Int $\mathbf{J}$ Radiat Oncol Biol Phys 2004;60:1419-24.

[40] Scarfone C, Laverly WC, Cmelak AJ, et al. Prospective feasibility trial of radiotherapy target definition for head and neck cancer using 3-dimensional PET and CT imaging. J Nucl Med 2004;45:543-52.

[41] Nishioka T, Shiga T, Shirato H, et al. Image fusion between 18 FDG-PET and MRI/CT for radiotherapy planning of oropharyngeal and nasopharyngeal carcinomas. Int $\mathbf{J}$ Radiat Oncol Biol Phys 2002;53:1051-7.

[42] Daisne J, Duprez T, Weynant B, et al. Impact of image coregistration with computed tomography (CT), magnetic resonance (MR), and positron emission tomography with fluorodeoxyglucose (FDG-PET) on delineation of GTV's in oropharyngeal, laryngeal and hypopharyngeal tumors. Int J Radiat Oncol Biol Phys 2002;54:15-6.

[43] Ciernik F, Dizendorf E, Baumert BG, et al. Radiation treatment planning with an integrated positron emission and computer tomography (PET/CT) A feasibility study. Int J Radiat Oncol Biol Phys 2003;52:853-63.

[44] Gourin CG, Williams HT, Seabolt WN, et al. Utility of positron emission tomography-computed tomography in identification of residual nodal disease after chemoradiation for advanced head and neck cancer. Laryngoscope 2006;116(5):705-10.

[45] Yao M, Smith RB, Graham MM, et al. The role of FDG PET in management of neck metastasis from head-and-neck cancer after definitive radiation treatment. Int $\mathbf{J}$ Radiat Oncol Biol Phys 2005;63:991-9.

[46] Andrade RS, Heron DE, Degirmenci B, et al. Post-treatment assessment of response using FDG-PET/CT for patients treated with definitive radiation therapy for head and neck cancers. Int J Radiat Oncol Biol Phys 2006;65(5):1315-22.

[47] Goerres GW, Schmid DT, Bandhauer F, et al. Positron emission tomography in the early follow-up of advanced head and neck cancer. Arch Otolaryngol Head Neck Surg 2004; 130:105-9.

[48] Porceddu SV, Jarmolowski E, Hicks RJ, et al. Utility of positron emission tomography for the detection of disease in residual neck nodes after (chemo)-radiotherapy in head and neck cancer. Head Neck 2005;27:175-81.

[49] Zimmer LA, McCook B, Meltzer C, et al. The use of combined PET/CT for localizing recurrent head and neck cancer: the Pittsburgh experience. Ear Nose Throat J 2005;84(2): $104,106,108-110$.

[50] Andrade RS, Heron DE, Degirmenci B, et al. Post-treatment assessment of response using FDG-PET/CT for patients treated with definitive radiation therapy for head and neck cancers. Int J Radiat Oncol Biol Phys 2006;65:1315-22.

[51] Ryan WR, Fee WE, Le QT, et al. Positron-emission tomography for surveillance of head and neck cancer. Laryngoscope 2005;115:645-50.

[52] Brkovich VS, Miller FR, Karnad AB, et al. The role of positron emission tomography scans in the management of the N-positive neck in head and neck squamous cell carcinoma after chemoradiotherapy. Laryngoscope 2006;116:855-8.

[53] Nayak JV, Walvekar RR, Andrade RS, et al. Deferring planned neck dissection after chemoradiation therapy in stage IV head and neck cancer: the utility of PET/CT. American head and neck society annual meeting \& research workshop on the biology, prevention and treatment of head and neck cancer, August 2006.

[54] Perlow A, Bui C, Shreve P, et al. High incidence of chest malignancy detected by FDG PET in patients suspected of recurrent squamous cell carcinoma of the upper aerodigestive tract. J Comput Assist Tomogr 2004;28(5):704-9. 
[55] de Visscher AV, Manni JJ. Routine long-term follow-up in patients treated with curative intent for squamous cell carcinoma of the larynx, pharynx, and oral cavity. Does it make sense? Arch Otolaryngol Head Neck Surg 1994;120(9):934-9.

[56] Leon X, Quer M, Diez S, et al. Second neoplasm in patients with head and neck cancer. Head Neck 1999;21(3):204-10.

[57] Helal BO, Merlet P, Toubert ME, et al. Clinical impact of (18)F-FDG PET in thyroid carcinoma patients with elevated thyroglobulin levels and negative (131)I scanning results after therapy. J Nucl Med 2001;42:1464-9.

[58] Nanni C, Rubello D, Fanti S, et al. Role of 18F-FDG-PET and PET/CT imaging in thyroid cancer. Biomed Pharmacother 2006;60(8):409-13.

[59] Palmedo H, Bucerius J, Joe A, et al. Integrated PET/CT in differentiated thyroid cancer: diagnostic accuracy and impact on patient management. J Nucl Med 2006;47(4):616-24.

[60] Szakall S Jr, Esik O, Bajzik G, et al. 18F-FDG PET detection of lymph node metastases in medullary thyroid carcinoma. J Nucl Med 2002;43(1):66-71.

[61] Bockisch A, Brandt-Mainz K, Gorges R, et al. Diagnosis in medullary thyroid cancer with $[18 F]$ FDG-PET and improvement using a combined PET/CT scanner. Acta Med Austriaca 2003;30:22-5.

[62] Schaefer NG, Hany TF, Taverna C, et al. Non-Hodgkin lymphoma and Hodgkin disease: coregistered FDG PET and CT at staging and restaging-do we need contrast-enhanced CT? Radiology 2004;232(3):823-9.

[63] Swetter SM, Carroll LA, Johnson DL, et al. Positron emission tomography is superior to computed tomography for metastatic detection in melanoma patients. Ann Surg Oncol 2002;9(7):646-53.

[64] Steinert HC, Huch Boni RA, Buck A, et al. Malignant melanoma: staging with whole-body positron emission tomography and 2-[F-18]-fluoro-2-deoxy-D-glucose. Radiology 1995; 195(3):705-9.

[65] Strobel K, Dummer R, Husarik DB, et al. High-risk melanoma: accuracy of FDG PET/CT with added CT morphologic information for detection of metastases. Radiology 2007; 244(2):566-74.

[66] Brun E, Ohlsson T, Erlandsson K, et al. Early prediction of treatment outcome in head and neck cancer with 2-18FDG PET. Acta Oncol 1997;36:741-7.

[67] Kitagawa Y, Sadato N, Azuma H, et al. FDG PET to evaluate combined intra-arterial chemotherapy and radiotherapy of head and neck neoplasms. J Nucl Med 1999;40: $1132-7$.

[68] Allal AS, Dulguerov P, Allaoua M, et al. Standardized uptake value of 2-[(18)F]fluoro-2-deoxy-D-glucose in predicting outcome in head and neck carcinomas treated by radiotherapy with or without chemotherapy. J Clin Oncol 2002;20:1398-404.

[69] Huang SC. Anatomy of SUV: standardized uptake value. Nucl Med Biol 2000;27:643-6.

[70] Nakamoto Y, Bennett B, Dara P, et al. Effects of nonionic intravenous contrast agents at PET/CT imaging: phantom and canine studies. Radiology 2003;227:817-24.

[71] Blodgett TM, Casagranda B, Townsend DW, et al. Issues, controversies, and clinical utility of combined PET/CT imaging: what is the interpreting physician facing? AJR Am J Roentgenol 2005;184(Suppl 5):S138-45. 